\title{
Emergency general surgery models in Australia: A cross-sectional study
}

\section{Ned Kinnear¹, Jennie Han², Minh Tran², Matheesha Herath², Samantha Jolly², Derek Hennessey ${ }^{3}$,} Christopher Dobbins ${ }^{1}$, Tarik Sammour ${ }^{1,2}$, and James Moore ${ }^{1,2}$

1. Department of Surgery, Faculty of Health and Medical Sciences, University of Adelaide, Adelaide, Australia

2. Department of Surgery, Royal Adelaide Hospital, Adelaide, Australia

3. Department of Urology, Mercy University Hospital, Cork, Ireland

\section{RESEARCH}

Please cite this paper as: Kinnear N, Han J, Tran M, Herath M, Jolly S, Hennessey D, Dobbins C, Sammour T, Moore J. Emergency general surgery models in Australia: A crosssectional study. AMJ 2019;12(11):303-311.

https://doi.org/10.35841/1836-1935.12.11.303-311

\section{Corresponding Author:}

Dr Ned Kinnear

Adelaide Medical School, University of Adelaide

North Terrace, Adelaide SA 5000, AUSTRALIA

Email: ned.kinnear@gmail.com

\section{ABSTRACT}

\section{Background}

Emergency general surgery (EGS) patients experience superior outcomes when cared for within an acute surgical unit (ASU) model. However, the EGS structures in most Australian hospitals remain unknown.

\section{Aims}

This study aimed to describe the national spectrum of EGS models.

\section{Methods}

Surgical staff were contacted in all Australian public hospitals of medium $(>2,000$ patient separations perannum) or greater peer group. Primary outcomes were incidence of each EGS model. Secondary outcomes were the relationship of EGS model to objective hospital variables, and qualitative reasons for choice of model. An ASU allocated a general surgeon solely to EGS patients for $\geq 50$ per cent of business hours. A Hybrid model did not have this feature, but provided either a doctor-in-training rostered solely to EGS for $\geq 50$ per cent of business hours, or $\geq 2$ protected theatre half-day EGS lists per week.
Results

One hundred and nineteen of 120 eligible hospitals participated (99 per cent). Sixty-four hospitals (54 per cent) reported utilising hybrid model. ASU implementation was significantly more common amongst hospitals of greater peer group $(p<0.0001)$, bed number $(p<0.0001)$, surgeon pool $(p=0.0003)$ and trauma service sophistication $(p=0.0002)$. Leading reported drivers for ASU commencement were aims to improve EGS patient care and decrease after-hours operating, while common barriers against ASU uptake were insufficient EGS patient load or surgeon on-call pool.

\section{Conclusion}

ASU or Hybrid models of care for EGS patients may be more widespread than currently reported. Introduction of such structures is heavily dependent on hospital and staff size, trauma subspecialisation and EGS patient throughput.

\section{Key Words}

Acute surgical unit, acute general surgery, acute care surgery, emergency general surgery

\section{What this study adds:}

\section{What is known about this subject?}

Australian hospitals remain free to choose their model of emergency general surgical care, and the structure in the great majority of centres remains unknown.

\section{What new information is offered in this study?}

This study enrolled 99 per cent of medium-or-larger Australian public hospitals. Most employed an ASU or hybrid model. Appeals included improving care and reducing night time operating.

3. What are the implications for research, policy, or practice?

This study provides guidance for policy makers, and for hospitals considering implementing an ASU. 


\section{Background}

General surgery is the surgical specialty of the gastrointestinal tract, thyroid gland, peripheral abscesses and trauma. In emergencies or rural locations, general surgery may also include procedures of the head, chest, skeleton and scrotum. Emergency patients comprise the majority of workload and deaths within general surgical departments. ${ }^{1}$ In the United States of America (USA), they annually comprise $>3$ million admissions at a cost of >USD28 billion. ${ }^{2,3}$ The number of emergency general surgery (EGS) patients is also rising relentlessly by $>30$ per cent each decade in Europe and the USA. ${ }^{4,5}$ However, despite representing the majority of general surgical workload, EGS patients rarely feature in hospital key performance indicators. ${ }^{6}$ Traditional structures have therefore long been aligned with providing optimum care for elective patients, often at the expense of EGS patients. Surgeons and trainees were typically rostered for elective operating and clinics while EGS patients were managed ad-hoc, either by delay or cancellation of elective patients, or after-hours.

In 1996, a new model was trialled with the separation of elective and emergency general surgical activity. ${ }^{5}$ This model would come to be best known as the acute surgical unit (ASU) and its positive results led to uptake across Europe, the USA and Australasia. ${ }^{7,8}$ The model's central component is a surgeon, on-site in business hours and oncall afterhours, dedicated to EGS patients without elective or private commitments during their shift. Additional features may include an EGS-allocated trainee and protected theatre access.

To date, a total of sixteen Australian public hospitals have published their uptake of the model, while another two have documented successful persistence with traditional or sub-specialty models (Supplementary Resource 1). However, for the great majority of public hospitals in Australia, the model in use remains unknown. In 2010, General Surgery Australia's (GSA) '12 Point Plan for Emergency General Surgery' recommended separation of emergency and elective activity. ${ }^{9}$ However, these were nonbinding recommendations and explicitly expected variation in EGS models. Departments remain free to choose their preferred system and no national registry or automated reporting of structure exists. This lack of information regarding EGS models in the large majority of Australian hospitals is problematic. A comprehensive national crosssectional study could be used to guide other hospitals considering commencing an ASU, or by health bodies to inform future policy.
Given the evidence of the ASU model's benefit but the opacity of its uptake in Australia, we aimed therefore to assess the structure of EGS care of adults in all Australian medium to major public hospitals. This included the chronological and geographical spread of EGS models, the impact of trauma care and other variables on choice of EGS model, and factors promoting and preventing ASU establishment in this country.

\section{Method}

\section{Sample frame}

In this cross-sectional study, Australian public hospitals offering elective general surgery were identified from Australian Institute of Health and Welfare's (AIHW) data, published December 2018. ${ }^{10}$ Small, children's, un-peered hospitals or private hospitals were excluded (see Supplementary Resource 2 for definitions).

\section{Recruitment methods}

After inter-researcher verbal rehearsal for standardisation, all medium- to major-sized Australian hospitals were contacted by telephone during March-April 2019. At each hospital, the on-call general surgery registrar and the head of general surgery (or available senior surgeon) were each invited to participate. Eligible registrars were any doctors responsible for EGS referrals on the day the authors contacted their hospital who had at least one tier of EGS oncall supervision above them, regardless of whether they were in accredited training programs or referred to as 'registrars' in their hospital. At least three attempts were made to contact each participant. Before delivery of the questionnaire, the voluntary and confidential nature of the study was clearly explained and verbal consent to participate was obtained.

\section{Data collection}

A pre-defined questionnaire (Supplementary Resource 3) was administered, with responses recorded in writing. No audio was recorded and incentives for participation were not used.

Primary outcomes were proportions of hospitals employing each EGS model and chronological and geographic spread of the ASU or Hybrid model. Secondary outcomes were the relationship of EGS model to trauma surgery care and other variables, reasons for and against model implementation and hospital-reported cost analyses.

Each hospital's structure of EGS delivery was categorised as ASU, Hybrid or Traditional. An ASU model was defined as one in which the on-call general surgeon was allocated to 
EGS patients for $\geq 50$ per cent of business hours. A Hybrid model did not allocate a dedicated on-call surgeon but did have either a doctor-in-training rostered solely to EGS patients for $\geq 50$ per cent of business hours, or $\geq 2$ protected theatre half-day lists per week for EGS patients. Traditional units had none of these features. Other structural features such as dedicated EGS beds or handover routines were noted but did not affect the assigned EGS model. Hospitals were asked to describe their handover practices when surgeons changed on-call, and the hospital's level of trauma care. Trauma care categories were Level 1 trauma centre, non-Level 1 hospitals still accepting major trauma or on bypass for major trauma.

To most accurately characterise a department's model, at each hospital both the on-call general surgery registrar and senior surgeon were asked to describe the elective commitments of both registrar and surgeon while on call, as well as the existence of protected EGS theatre access. In cases of conflicting data, the surgeon's response was taken as correct, except regarding registrar commitments, where the registrar's lived experience was prioritised. When graphing the number of ASU or Hybrid units over time, a simple sigmoid growth curve was created between the known start (no such model pre-2005 ${ }^{11}$ ) and end points (total such units at time of study). Ethics approval was granted by the Central Adelaide Local Health Network human research ethics committee (R20180812).

\section{Statistical analysis}

Categorical measures were summarized as proportions and assessed with Pearson's chi-square test. All tests were twotailed and significance was assessed at the 5 per cent alpha level. Missing data was reported if present.

\section{Results}

\section{Primary outcomes}

Two hundred and forty-three public hospitals were found to offer elective general surgery in Australia. After excluding 109 small, children's or un-peered sites, the remaining 134 medium to major sized hospitals were contacted. Respondents identified one additional site introduced subsequent to AlHW list publication, resulting in 135 hospitals (Supplementary Resource 4). Fifteen reported neither admitting nor operating on emergency patients, and were excluded from further analysis, resulting in 120 total eligible hospitals. From these, the questionnaire was completed by at least one staff member at 119 (99 per cent) sites, including $107 / 120$ surgeons (89 per cent), and 115/116 registrars (99 per cent), with four sites not involving registrars in EGS on-call (Table 1).
The implementation of ASU or Hybrid units was observed to grow steadily from 2005 to present, with an ASU or Hybrid model now present in all states and territories, and the majority of medium to major Australian public hospitals (Figures 1-2). Thirty-three hospitals (28 per cent) utilised an ASU model, while a further 31 (26 per cent) chose a Hybrid model. The remaining 55 hospitals ( 46 per cent) employed a Traditional model. This included four hospitals with a subspecialty model, whereby all general surgical sub-specialty teams are on call Monday - Friday (and also the weekend, in some cases) for patients with relevant diagnoses.

Of enrolled hospitals, 113/119 (95 per cent) reported the presence or not of handover practices when the surgeon on-call changed. Twenty-seven hospitals described established departmental rules for handover at these times. In all cases, these rules were for patients still requiring an operation, with ongoing diagnostic ambiguity and/or remaining admitted after a pre-specified duration. Such rules were significantly more likely to occur within an ASU, being present in 15/33 such units (45 per cent), 8/31 Hybrid models (26 per cent) and 4/49 Traditional models ( 8 per cent) $(p=0.0005)$.

\section{Secondary outcomes}

ASU models were significantly more common in hospitals of greater peer group $(p<0.0001)$, greater bed number $(p<0.0001)$ or with more surgeons in the EGS on-call pool $(p=0.0003)$ (Figure 2). Put simply, hospitals that were larger or metropolitan were more likely to incorporate this structure than medium sized or regional centres. Of 33 hospitals utilising an ASU model, none were in mediumsized regional hospitals, only two were in hospitals of $\leq 150$ beds and just one in a hospital with $\leq 4$ surgeons in the EGS on-call roster. EGS model was not related to Australian state or territory $(p=0.42)$.

Trauma care model was determined for $109 / 119$ sites (92 per cent), with ASU models significantly more common amongst hospitals provisioning more advanced trauma care $(p=0.002)$. Data on the presence of dedicated trauma surgeons was available for $106 / 119$ sites (89 per cent). Only seven sites reported having a dedicated trauma surgeon on staff. These were all within recognised Level 1 trauma centres, in hospitals with either ASU (six sites) or Hybrid models of EGS care (one site).

\section{Drivers for change}

Senior surgeons at 34/64 sites (53 per cent) with an ASU or Hybrid EGS model described their hospital's reasons for change from the Traditional model. Multiple causes were 
permitted per site. A total of 74 responses fitting fifteen categories were received (Supplementary Resource 5). The leading reported drivers for change were the desire to improve care for EGS patients (twelve hospitals), reduce after-hours operating (eleven hospitals) and address rising EGS patient load (nine). Notably, surgeons also reported ASU implementation in response to publicised benefits or governmental/ specialty-group reports. These included convincing results from the Prince of Wales Hospital, NSW, or Fremantle Hospital, WA, influential presentations by Past Presidents of the Royal Australasian College of Surgeons (RACS), GSA's aforementioned policy ${ }^{9}$ and the Honorary Peter Garling's commissioned inquiry into acute care services in New South Wales. ${ }^{12}$

\section{Barriers to change}

Conversely, 56/86 hospitals (65 per cent) employing Traditional or Hybrid EGS models reported considering changing further towards an ASU structure, but not doing so due to perceived barriers. Multiple causes were permitted per site. 68 responses fitting nine categories were received (Supplementary Resource 5). The leading reported barriers to instituting an ASU were insufficient EGS patient load (33 hospitals), insufficient surgeons to allocate one solely to EGS (eleven) and insufficient funding (nine). Three hospitals reported unsuccessful formal attempts to commence an ASU, all frustrated by insufficient funding. These occurred in one each of a large regional, medium metropolitan and large metropolitan hospital.

\section{ASU units of the past or future}

Included in the above 56 hospitals, twelve sites (six with Traditional model, six with the Hybrid model) reported planning to start an ASU in the future. Motivations were similar to those in 'Drivers for change', above. Separately, five hospitals reported trialling an ASU model but experiencing challenges and reverted to a Traditional (one site) or Hybrid model (four).

These are summarised in Supplementary Resource 6 . Reported reasons for reversion formed common themes of insufficient EGS load, insufficient staff surgeons and surgeon fatigue.

\section{Cost analyses}

Three of the 33 hospitals utilising an ASU reported assessing the financial impact of its introduction. Savings occurred in one major hospital and one large regional hospital, with the latter publishing their costings, ${ }^{13}$ while another large regional hospital experienced cost neutrality. Only $1 / 31$ hospitals with a Hybrid structure described financial assessment of the change in EGS structure. This major hospital observed that staffing in the new model required increased expenditure.

\section{Discussion}

These results suggest significant change during the last two decades in EGS care in Australia. This began with the establishment of the nation's first ASU in $2005^{11}$ and leads to present day, with the majority of medium to major public hospitals now utilising an ASU or Hybrid model. While this study's generalizability is limited by its assessment of only Australian centres, the identified leading motivators for EGS model change, namely improving patient care and reducing after-hours operating, are common globally. In Australia, national emergency access targets have been introduced in the last decade in an attempt to limit patient time in the emergency department to $<4$ hours. ${ }^{14}$ In this study, this directive was also frequently reported to have influenced ASU introduction (Supplementary Resource 5).

Several previous studies have attempted to establish the spectrum of EGS models. Via unspecified correspondence methods, Uranues et al., contacted 51 EGS 'experts' in 27 European countries. ${ }^{15}$ Receiving responses from 18 countries, three (17 per cent) were found to employ an ASU model. However, as demonstrated by our study and others, ${ }^{16}$ significant variation in EGS model exists within the same state or country and thus these results are only a guide to European practices. State-based assessments of hospitals in Canada, the USA and the United Kingdom (UK) have reported ASU uptake rates of 8-29 per cent. ${ }^{16-18}$ At the national level, a postal and email assessment of all American hospitals containing both an emergency department and operating theatre reached 1690/2811 (60 per cent) hospitals, with 16 per cent employing an ASU model. $^{2}$ Similarly, a UK online questionnaire enrolled $\geq 1$ colorectal surgeon from 104/135 (77 per cent) acute nonspecialist National Health Service Trusts, and reported ASU uptake in 26 per cent of hospitals. ${ }^{19}$ Taken together, ASU implementation rates in developed nations range from 8-29 per cent, similar to our findings. In comparison to other works, this study enjoyed an excellent response rate of 99 per cent. Furthermore, while most studies dichotomously categorised EGS structure to Traditional or ASU, this analysis allowed for real-world variety by including Hybrid structures. Additionally, this study's approach of categorising EGS structure based on staff rostering and theatre allocation, rather than hospital self-description, avoids confounding by the acknowledged practice whereby surgical departments re-brand without substantially modifying services. ${ }^{20}$ 
There has been limited interest in the last five years in an alternative EGS model; the Surgical Assessment Unit. ${ }^{21}$ None have been reported in Australia. These roster an EGSdedicated surgeon in the emergency department, to whom patients with probable EGS diagnoses are referred by the emergency department triage nurse (or in some countries, general practitioners). Patients bypass assessment by the emergency department. This structure may offer similar benefits to the ASU, although results have been mixed. Two hospitals in this study reported local interest, but not yet commencement, of a Surgical Assessment Unit. Both sites were stimulated by hospital renovations creating additional space in the emergency department.

Patient handover is a common cause of preventable injury in surgery, with emergency patients particularly at risk. ${ }^{22}$ Patient handover is infrequent within Traditional structures, with patients typically cared for by a single surgeon throughout their admission. Through increased consultant involvement and reduced after-hours operating, the ASU model potentially improves patient safety. However, shift work systems disrupt continuity of care and potentially worsens safety. Reassuringly however, the pooled effect of these factors appears positive, with all comparative ASU studies reporting equivalent or reduced complications. ${ }^{7}$ An Australian ASU study found that surgeon-to-surgeon handover did not affect patient outcomes, ${ }^{23}$ while in the UK, ASU trainees reported greater satisfaction with handover practices. $^{24}$ Similarly, our study found that standardised patient handover was more likely to occur in ASU models. However, such rules' existence in only 45 per cent of ASUs was lower than expected, given the model's need for frequent handover. Structured handovers in general surgery are known to significantly increase the quality of information transfer. ${ }^{25}$ Hospital departments caring for EGS patients, particularly within an ASU model, should examine and define their handover protocols.

Limitations of this study include its reliance on descriptions from participating doctors, who may be under time pressure or new in their position. There were occasional instances where surgeons and registrars differed in describing their EGS model. Our resolution approach described in the Methods section may nevertheless have rarely misallocated EGS model.

\section{Conclusion}

ASU or Hybrid models of care for EGS patients may be more widespread than currently reported. Introduction of such structures is heavily dependent on hospital and staff size and trauma subspecialisation, with no single model expected to fit all centres. These findings may provide guidance for policy makers and hospitals considering implementing an ASU.

\section{References}

1. Association of Surgeons of Great Britain and Ireland. Emergency General Surgery. London, England: Royal College of Surgeons; 2013.

2. Daniel VT, Ingraham AM, Khubchandani JA, et al. Variations in the Delivery of Emergency General Surgery Care in the Era of Acute Care Surgery. Jt Comm J Qual Patient Saf. 2019;45(1):14-23. doi: 10.1016/j.jcjq.2018.04.012

3. Ogola GO, Gale SC, Haider A, et al. The financial burden of emergency general surgery: National estimates 2010 to 2060. J Trauma Acute Care Surg. 2015;79(3):444-8. doi: 10.1097/TA.0000000000000787

4. Gale SC, Shafi S, Dombrovskiy VY, et al. The public health burden of emergency general surgery in the United States: A 10-year analysis of the Nationwide Inpatient Sample--2001 to 2010. J Trauma Acute Care Surg. 2014;77(2):202-8. doi: 10.1097/TA.0000000000000362

5. Addison PDR, Getgood A, Paterson-Brown S. Separating elective and emergency surgical care (The Emergency Team). Scott Med J. 2001;46(2):48-50.

6. Victorian Government Dept of Health. Good practice in management of emergency surgery: a literature review Melbourne, Australia. : Victorian Government; 2010 [Accessed 01/08/18]. Available from: https://www2.health.vic.gov.au/Api/downloadmedia/\% 7BA0B3F2D4-BEC7-4AC9-8DB5-61861ECCDE3A\%7D.

7. Nagaraja V, Eslick GD, Cox MR. The acute surgical unit model verses the traditional "on call" model: a systematic review and meta-analysis. World J Surg. 2014;38(6):1381-7. doi: 10.1007/s00268-013-2447-1

8. Bazzi ZT, Kinnear N, Bazzi CS, et al. Impact of an acute surgical unit on outcomes in acute cholecystitis. ANZ J Surg. 2018;88(12):E835-E9. doi: 10.1111/ans.14802

9. General Surgery Australia. 12 Point Plan General for Emergency General Surgery. Melbourne, Australia: Royal Australasian College of Surgeons; 2010 [Accessed 01/08/18]. Available from: https://www.generalsurgeons.com.au/media/files/Publ ications/PLN 2010-09-19 GSA 12 Point Plan.pdf.

10. Australian Institute of Health and Welfare. Elective surgery waiting times 2017-18: Australian hospital statistics. Canberra, Australia: AlHW; 2018 [Accessed 10/12/18]. Available from: https://www.aihw.gov.au/reports/hospitals/electivesurgery-waiting-times-2017-18/contents/table-of- 
contents.

11. Lien I, Wong SW, Malouf $P$, et al. Effect of handover on the outcomes of small bowel obstruction in an acute care surgery model. ANZ J Surg. 2014;84(6):442-7. doi: 10.1111/j.1445-2197.2012.06248.x

12. Garling P. Final Report of the Special Commission of Inquiry; Acute Care Services in NSW Public Hospitals Sydney, Australia: NSW Government, Dept of Premier \& Cabinet; 2008 [Accessed 01/08/18]. Available from: https://www.dpc.nsw.gov.au/publications/specialcommissions-of-inquiry/special-commission-of-inquiryinto-acute-care-services-in-new-south-wales-publichospitals/.

13. King $M$. Implementing an acute surgical unit and its affect on NEST targets at Wagga Wagga Health Service [Internet]. New South Wales Agency for Clinical Innovation. 2013.

14. Perera ML, Roberts MJ, Gnaneswaran N, et al. The 'Four-hour target' (NEAT) and the impact on metropolitan acute urological services. BJU International. 2015;115(Supp 4):78.

15. Uranues S, Lamont E. Acute care surgery: The European model. World J Surg. 2008;32(8):1605-12. doi: 10.1007/s00268-008-9501-4

16. Bandy NL, DeShields SC, Cunningham TD, et al. Statewide assessment of surgical outcomes and the acute care surgery model. J Surg Res. 2017;220:25-9. doi: 10.1007/s00268-008-9501-4

17. Joos E, Trottier V, Thauvette D. Interest and applicability of acute care surgery among surgeons in Quebec: a provincial survey. Can J Surg. 2013;56(4):E63-7.

18. Barthelmes L, Kakkilaya $H$, Jenkinson LR. An audit of the management of emergency surgical admissions in Wales: Are we keeping pace with the trend? Clinical Governance. 2004;9(1):31-3.

19. Symons NRA, McArthur D, Miller A, et al. Emergency general surgeons, subspeciality surgeons and the future management of emergency surgery: results of a national survey. Colorectal Dis. 2019;21(3):342-8. doi: 10.1111/codi.14474

20. Collins CE, Pringle PL, Santry HP. Innovation or rebranding, acute care surgery diffusion will continue. J Surg Res. 2015;197(2):354-62. doi: 10.1016/j.jss.2015.03.046

21. Fearon N, Nic An Riogh A, Silvio-Esteba L, et al. Ahead of the curve: Implementation of the first twenty-four hour acute surgical assessment unit (ASAU) in Ireland. Int J Surg. 2016;36 (Supplement 1):S71-S2.

22. Greenberg CC, Regenbogen SE, Studdert DM, et al. Patterns of communication breakdowns resulting in injury to surgical patients. J Am Coll Surg. 2007;204(4):533-40.

23. Lien I, Wong SW, Malouf $P$, et al. Effect of handover on the outcomes of small bowel obstruction in an acute care surgery model. ANZ J Surg. 2014;84(6):442-7. doi: 10.1111/j.1445-2197.2012.06248.x

24. Kennedy R, Kelly S, Grant S, et al. Northern Ireland General Surgery Handover Study: surgical trainees' assessment of current practice. Surgeon. 2009;7(1):103.

25. Jones HG, Watt B, Lewis L, et al. Structured Handover in General Surgery: An Audit of Current Practice. J Patient Saf. 2019;15(1):7-10. doi: 10.1097/PTS.0000000000000201.

\section{ACKNOWLEDGEMENTS}

This research was supported by the University of Adelaide and its Adelaide Graduate Centre.

\section{PEER REVIEW}

Not commissioned. Externally peer reviewed.

\section{CONFLICTS OF INTEREST}

The authors declare that they have no competing interests.

\section{FUNDING}

Ned Kinnear received a University of Adelaide Divisional Scholarship, a Hospital Research Foundation post-graduate scholarship and a National Health and Medical Research Council post-graduate scholarship in relation to this work. However, no funders influenced the study, nor had access to the manuscript or its results prior to publication.

\section{ETHICS COMMITTEE APPROVAL}

Ethics approval was granted by the Central Adelaide Local Health Network human research ethics committee (R20180812). 
Figure 1: Number of Acute Surgical Unit or Hybrid emergency general surgery models in Australia

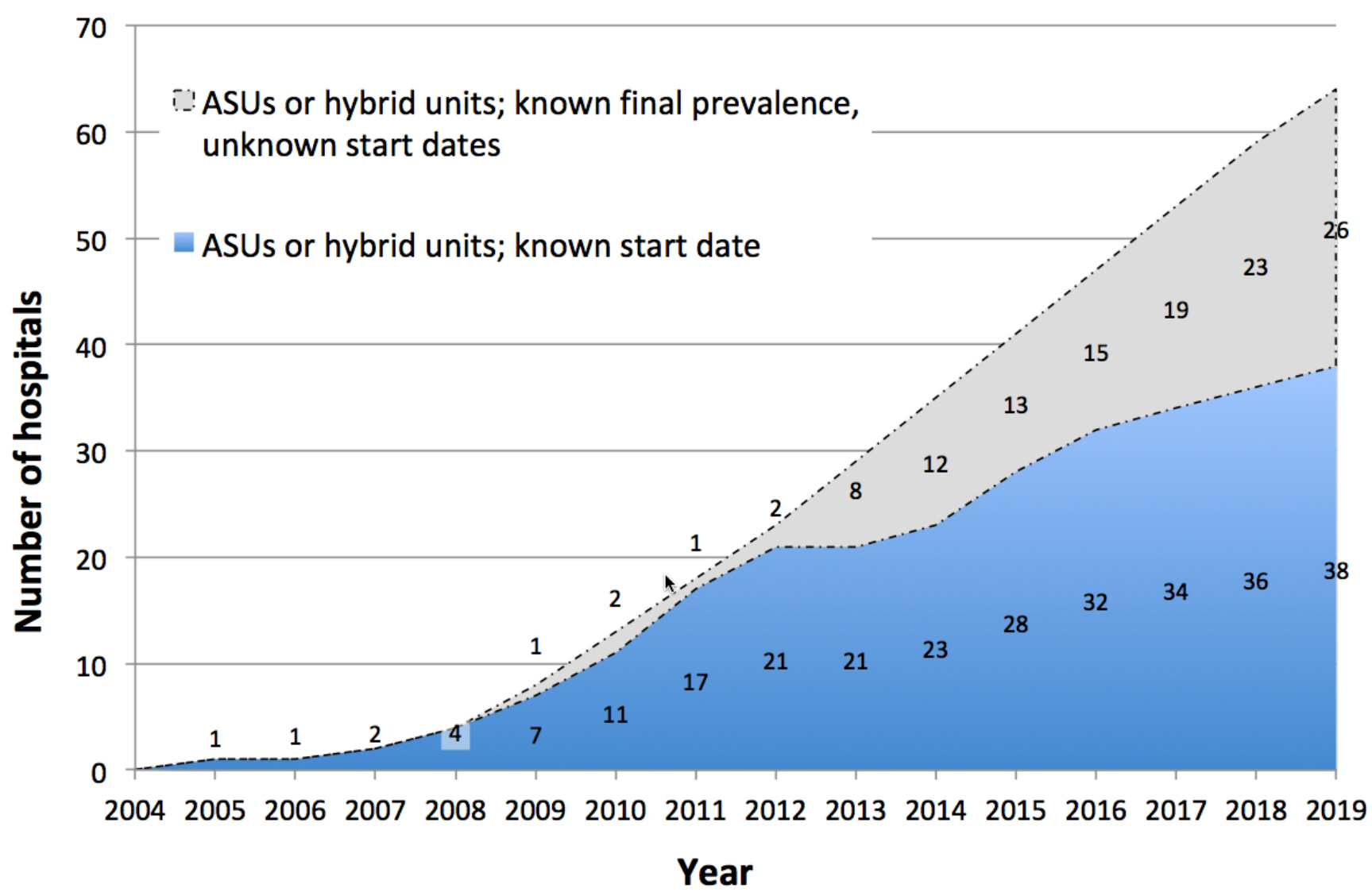


Figure 2: Distribution of emergency general surgery models by (A) hospital peer group, (B) hospital bed size, (C) number of surgeons in on-call pool, (D) Australian state or territory, (E) major trauma model and (F) entity receiving referrals overnight
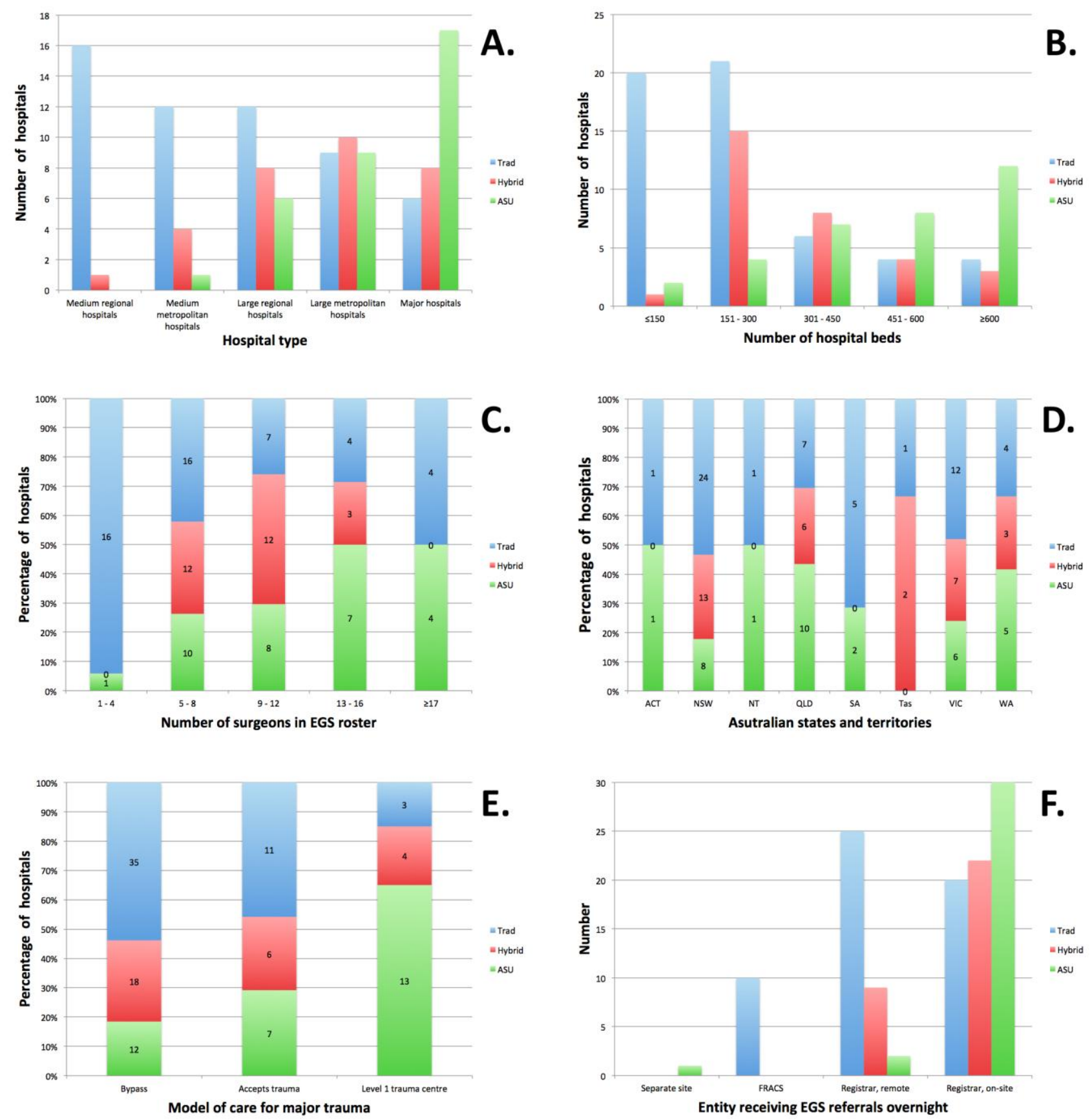

Legend

ASU: acute surgical unit. EGS: emergency general surgery. FRACS: Fellow of the Royal Australasian College of Surgeons. Trad: traditional.

NB.1. Data was available for $119 / 119$ (100\%) hospitals for all graphs except Figures 2.C. and 2.E., for which data was available for only 104/119 (87\%) and 109/119 (92\%) sites, respectively. 
Table 1: Cross-sectional study results by staff and service

\begin{tabular}{|c|c|c|c|}
\hline \multicolumn{2}{|l|}{ Staff or service } & \multicolumn{2}{|c|}{ Number (\%) } \\
\hline \multicolumn{2}{|c|}{$\begin{array}{l}\text { Registrars } \\
\text { Enrolled/ Declined/ Uncontactable }\end{array}$} & \multicolumn{2}{|c|}{$115 / 1 / 0+$} \\
\hline \multicolumn{2}{|l|}{$\begin{array}{l}\text { Consultants } \\
\text { Enrolled/ Declined }\end{array}$} & \multicolumn{2}{|c|}{$107 / 3 / 10$} \\
\hline $\begin{array}{l}\text { EGS model } \\
\text { Acute surgical unit } \\
\text { Hybrid } \\
\text { Traditional } \\
\text { Unknown }\end{array}$ & $\begin{array}{l}n(\%) \\
n(\%) \\
n(\%) \\
n(\%)\end{array}$ & $\begin{array}{l}33 \\
31 \\
55 \\
1\end{array}$ & $\begin{array}{l}(28 \%) \\
(26 \%) \\
(46 \%) \\
(1 \%)\end{array}$ \\
\hline $\begin{array}{l}\text { Services dedicated to } \\
\text { On-call surgeon } \\
\text { On-call registrar } \\
\text { Theatre } \\
\text { Beds, any } \\
\text { No. of EGS beds } \\
\end{array}$ & $\begin{array}{l}\text { tients } \\
n(\%) \ddagger \\
n(\%) \ddagger \\
n(\%) \S \\
n(\%) \\
\text { median (range) }\end{array}$ & $\begin{array}{l}33 \\
49 \\
82 \\
15 \\
12\end{array}$ & $\begin{array}{l}(28 \%) \\
(41 \%) \\
(69 \%) \\
(13 \%) \\
(4-28)\end{array}$ \\
\hline 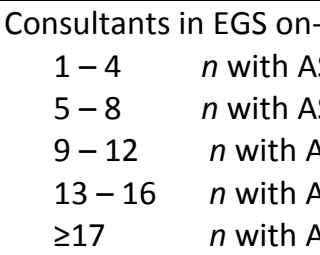 & $\begin{array}{l}\text { ster } \\
\text { tal (\%) } \\
\text { tal (\%) } \\
\text { tal (\%) } \\
\text { tal (\%) } \\
\text { tal (\%) }\end{array}$ & $\begin{array}{rr}1 / & 17 \\
10 / & 38 \\
8 / & 27 \\
7 / & 14 \\
4 / & 8\end{array}$ & $\begin{array}{l}(6 \%) \\
(26 \%) \\
(30 \%) \\
(50 \%) \\
(50 \%)\end{array}$ \\
\hline
\end{tabular}

ASU: acute surgical unit. N: number. + : four sites reported not involving registrars in EGS on-call. ¥: allocated to EGS patients for $\geq 50 \%$ of business hours. $\S: \geq 2$ protected theatre lists per week for EGS patients. \%: percentage. Denominators for all percentages are the 119 participating hospitals, unless otherwise specified 